\title{
La prise en compte du contexte macro-économique mondial : l'approche par macro-scénarios
}

\section{Taking into account the world macro-environment in macro-scenarios}

Oléagineux, Corps Gras, Lipides. Volume 9, Numéro 5, 346-51, Septembre - Octobre 2002, Dossier : Prospective et recherche agronomique

Auteur(s) : Antoine MESSEAN, Pierre GONOD, Yves DRONNE, Hélène LECCEUR, Michel SEBILLOTTE, INRA, DADP, 147, rue de I'Université, 75338 Paris cedex 07, France.

Author(s) : Antoine MESSEAN, Pierre GONOD, Yves DRONNE, Hélène LECEUU, Michel SEBILLOTTE

Résumé : Dans la méthode prospective SYSPAHMM, élaborée à I'INRA, nous construisons des microscénarios du futur à partir d'une description fouillée de la situation d'état du système étudié. Pour cela nous commençons par considérer que l'environnement socio-économique et écologique du système étudié est stable. Or, nous savons que cela est faux. Pour remédier à ce problème et sur l'exemple des protéines végétales et animales, nous avons, à partir d'hypothèses sur les modes de gouvernance mondiale, construit des macroscénarios du monde qui se caractérisent par six ensemble de variables. Nous étudions alors leurs effets sur le devenir des microscénarios et réciproquement. De cette manière, il est possible, au bout de quelques années, de comprendre le sens d'évolution générale du système étudié et, en actualisant les hypothèses qui ont servi à construire les microscénarios, de réécrire, sans recommencer tout le travail de prospective, des microscénarios plus adaptés à leur environnement. Celui-ci est décrit par le macroscénario dont la situation mondiale s'est rapprochée. L'exemple des protéines permet d'illustrer la démarche.

Summary : In the SYSPAHMM foresight method designed by INRA, a detailed analysis of the actual functioning of the system under consideration led to the elaboration of various future patterns, socalled micro-scenarios. In such an approach, the overall socio-economic macro-environment is considered to be stable over a rather long period of time. Such an hypothesis is misleading. In order to refine the method, global scenarios of the world macro-environment were defined during a case study on protein prospects. These so-called macro-scenarios have been built by using various hypotheses about some features, such as the kinds of governance and economic growth as well as the role of environmental issues in world development. Their influence on the micro-scenario behaviour and the effect of each micro-scenario on the plausibility of the macro-scenarios have been assessed. This leads to a better understanding of the overall evolution of the system. Moreover, it allows for an easy update of the initial set of hypotheses and the subsequent micro-scenarios depending on the specific macro-environment that is envisioned.

\section{ARTICLE}

Dans le cadre de l'exercice de prospective « Les protéines végétales et animales. Enjeux de société et défis pour l'agriculture et la recherche " [1], la démarche générale de la méthode DADP a été appliquée. À partir d'une description fouillée de la situation d'état du système des protéines qui 
permet d'identifier les processus à l'œuvre, la mise en dynamique du système s'effectue en formulant des hypothèses sur ces processus (poursuite du processus, rupture du processus, émergence de processus nouveaux), puis en construisant, à partir de ces hypothèses, des scénarios d'évolution. Ceuxci permettent alors de formuler des stratégies possibles pour les différents acteurs concernés et d'éclairer les décideurs sur les choix qu'ils ont à faire.

Dans le cas des protéines, environ deux cents hypothèses d'évolution du système ont été retenues par la cellule d'animation et les groupes d'experts. Comme la question des protéines est très large et aborde des domaines très divers, de nombreuses hypothèses portent sur le contexte géopolitique, économique et agricole à l'échelle mondiale et ne sont pas spécifiques au secteur des protéines.

Afin d'éviter de relever le défi d'une prospective très large sur l'agriculture mondiale dans son contexte économique, nous avons décidé de structurer notre prospective de telle façon que le cadrage macroéconomique mondial soit pris en compte mais en faisant en sorte que l'exercice de prospective proprement dit - la construction de micro-scénarios et l'analyse des conséquences en termes de stratégies de recherche - soit bien articulé autour du questionnement sur les protéines à l'échelle européenne.

Nous avons ainsi identifié un sous-ensemble de soixante-dix-neuf hypothèses directement liées aux processus du système européen des protéines dont nous avons analysé la dynamique selon la méthodologie DADP, en analysant les relations entre hypothèses, en constituant des agrégats d'hypothèses et en construisant des micro-scénarios à partir de ces agrégats (cf. dans le même numéro l'article sur la méthode SYSPAHMM, M. Sebillotte et C. Sebillotte).

En ce qui concerne le contexte mondial et ses évolutions potentielles, nous avons choisi d'en élaborer quelques visions différenciées à moyen terme que nous appelons macro-scénarios, en vue d'apprécier la sensibilité du système européen des protéines aux grandes évolutions potentielles du contexte socio-politique.

Ces macro-scénarios mondiaux sont ainsi une structuration définissant a priori un certain nombre d'« images contrastées " de l'environnement mondial à partir d'hypothèses sur les règles du jeu international, la croissance démographique, le développement économique, les modèles de consommation et les systèmes de production. Il s'agit en fait de pouvoir confronter les anticipations du système européen des protéines à ces images contrastées de futurs possibles pour l'environnement géopolitique mondial. On ne s'intéresse donc ni à la probabilité de réalisation de chacun de ces macroscénarios, ni à leur déclinaison précise, mais à leur cohérence interne et à leur capacité à balayer un champ aussi large que possible de situations mondiales contrastées. C'est cette démarche générale d'élaboration des macro-scénarios que nous décrivons dans cet article.

\section{La construction des macro-scénarios}

À côté de quelques hypothèses implicites, comme l'absence de catastrophes naturelles ou le maintien d'un système de marché, sous une forme ou une autre, nous avons explicité trois grandes hypothèses :

* la croissance démographique évoluera selon les prévisions actuelles de l'ONU et ne sera donc pas l'objet d'hypothèses contrastées ;

* il n'y aura pas d'effet majeur de l'évolution du climat sur la disponibilité en ressources naturelles ${ }^{3}$; 
* I'énergie restera relativement bon marché dans le cadre de notre horizon temporel.

Ces grandes hypothèses ne seront pas remises en cause dans l'élaboration de ces macro-scénarios.

\section{La structuration des macro-scénarios}

La structuration a priori des macro-scénarios s'est effectuée en retenant comme clé principale le type de gouvernance qui pourrait s'imposer au niveau mondial : ultra-libéralisme, gouvernance mondiale ou gouvernance régionale ${ }^{4}$. Chacun de ces types coexistent partiellement aujourd'hui, mais nous avons choisi de les extrémiser pour construire nos macro-scénarios.

La première clé de structuration des macro-scénarios de cadrage porte sur une accentuation forte du type de gouvernance dans trois directions :

- un ultralibéralisme dominé par des grandes firmes multinationales avec le soutien des États occidentaux parmi lesquels les États-Unis ont un poids déterminant. Le marché sans entrave favorise la croissance et l'esprit d'entreprise, ressort du capitalisme. La modernisation et la croissance favorisent l'apparition et l'essor des classes moyennes, qui, en retour, se sentent solidaires du développement démocratique, la liberté économique allant de pair avec la liberté politique. Le jeu social est à somme positive ;

- une gouvernance mondiale, domaine du multilatéralisme, dans laquelle les organisations intergouvernementales (OIG) telles que I'ONU, la FAO, le BIT, I'OMS, le PNUD, le FMI, la Banque mondiale, I'OMC ou l'OCDE reçoivent des mandats étendus de la part des gouvernements nationaux et disposent d'une grande liberté d'action, même si elle reste contrôlée. Il faut donc qu'il y ait convergence des politiques nationales pour qu'un mandat prenne corps. Certaines catastrophes peuvent évidemment provoquer une telle convergence, mais également la prise de conscience des risques et l'émergence de nouvelles valeurs (exemple : la prise de conscience des problèmes de l'environnement par I'opinion publique) ;

- des gouvernances régionales dans lesquelles les champs d'activité sont généralement circonscrits au domaine économique, à l'émergence et au renforcement de zones économiques coordonnées. Les OIGR (organisations intergouvernementales régionales) ont des finalités variables : I'ALENA vise essentiellement la libéralisation commerciale, le MERCOSUR se propose de développer les échanges à I'intérieur de la zone, l'UE a des objectifs qui dépassent l'économie et le commerce et constituent, avec la monnaie unique, les prémices d'une intégration sociale. Dans le système mondial, ces OIGR sont à la fois partenaires et concurrentes, la négociation principale se nouant entre I'ALENA et I'UE. Les OIGR peuvent soit constituer des digues face au libéralisme, soit, au contraire, favoriser ce dernier.

Par ailleurs, pour chaque type de gouvernance, nous avons distingué deux variantes en prenant en compte une clé secondaire de structuration - croissance économique, prise en compte du développement durable et importance des marchés - qui permettent d'élaborer au total six macroscénarios, longuement décrits dans [1] :

- Dans le cas de l'ultralibéralisme, nous avons distingué une première variante dans laquelle on observerait une forte croissance économique des principaux pays (UL1), notamment les pays asiatiques, ce qui signifierait, entre autres, une forte capacité à l'importation de produits agricoles et alimentaires. Le " tout marché " entraîne un recul des États et une diminution de leurs rôles. Une 
deuxième variante avec une hypothèse de croissance beaucoup plus faible (voire de récession), notamment pour les zones asiatiques et la Russie, ou encore l'Amérique latine (UL2), entraîne de proche en proche une crise systémique mondiale.

- Dans le cas de la gouvernance mondiale, nous avons retenu une première variante dans laquelle cette gouvernance est essentiellement fondée sur des critères économiques et monétaires (GM1) ce qui irait, par exemple, dans le sens d'une domination du Fonds monétaire international (stabilisation des parités des monnaies, assurance d'une certaine croissance économique, aucune intervention au niveau réglementaire et au niveau environnemental). La seconde variante correspond au scénario d'un nouveau système de développement ou d'un " autre développement " (GM2) où les notions de durabilité et d'environnement sont directement prises en charge par des organisations internationales qui pourraient, entre autres, être rattachés à la FAO.

- Dans le cas des gouvernances régionales, nous avons retenu une première variante dans laquelle l'économie mondiale (et en particulier les marchés des grands produits agricoles) sont cogérés par deux superpuissances mondiales

- I'ALENA et l'UE - fortement intégrée chacune, qui assurent croissance économique et ouverture vers les autres grands regroupements de pays du monde (GR1). La seconde variante des gouvernances régionales correspond à une situation où l'UE et I'ALENA se replient sur leurs marchés intérieurs et limitrophes, engendrant une crise mondiale et un retour généralisé à l'autarcie (GR2).

Cette construction aboutit donc à un ensemble de six macro-scénarios regroupés dans le tableau 1.

\section{Les acteurs des macro-scénarios}

Si tous les acteurs sociaux sont présents dans les macro-scénarios, leurs influences varient selon le cadre qu'ils subissent ou qu'ils ont contribué à créer. Nous décrivons brièvement ci-après le positionnement des acteurs pour chacun des macro-scénarios.

- Le tout-libéral (UL1). Les firmes multinationales (FMN) sont les promotrices et les agents de I'ultralibéralisme. Les États nationaux occidentaux sont en alliance avec elles pour libérer les échanges et déréguler l'économie et les relations sociales. Les organismes intergouvernementaux mondiaux (OIG) sont sous influence forte dans ce cadre. Les organismes intergouvernementaux régionaux (OIGR) le sont aussi et d'autant plus fortement qu'ils sont proches de l'épicentre américain. Les organisations non-gouvernementales (ONG) sont un élément de contestation qui n'influe pas sur les grandes décisions. Les organisations sociales non gouvernementales (OSNG), qui comprennent, outre les ONG, les partis politiques, les syndicats et d'autres associations de la société civile, sont marginalisées. Le sentiment d'impuissance vis-à-vis des forces motrices du système, l'idéologie de l'irréversibilité appuyée par l'évolution technologique, conduisent au fatalisme et au déclin du politique. La concurrence exacerbée, les restructurations, le chômage de masse, affaiblissent les syndicats de travailleurs. Leurs luttes pour le maintien de l'emploi et des avantages acquis sont considérées par l'idéologie de l'ultralibéralisme comme un combat d'arrière-garde.

- La régulation mondiale (GM1). Les FMN restent présentes au premier plan mais elles ne sont plus seules. Les OIG renforcent leurs activités. Celles-ci ne sont pas un corps indépendant des États nationaux qui leur fixent leurs mandats et votent leurs moyens. Toutefois, elles peuvent constituer une 
force de propositions entraînante pour les États membres. Les autres acteurs des OSNG sont à l'arrière-plan de leurs États respectifs.

- La coopération entre grandes zones (GR1). Le positionnement des acteurs est assez proche de celui de la gouvernance mondiale GM1. Évidemment, les OIGR prennent plus d'importance. Celle-ci dépend du poids de la région considérée dans l'économie et la politique mondiales et aussi de la manière dont la région, " unité active » [3], crée son propre espace d'opération. Celui de l'ALENA est activé par la puissance des États-Unis, et la visée est l'expansion mondiale. L'espace d'action européen est le seul, avec celui du Japon, à présenter des intersections avec le grand espace américain. Ces intersections sont des zones de conflit ou de coopération. Dans celui-ci les États européens mettent en première ligne les représentants bruxellois, dont l'espace décisionnel s'accroît d'autant plus que le jeu international est difficile. Cette action est à la longue possible seulement si l'opinion publique et ses OSNG apportent leur soutien ${ }^{5}$. Si la construction européenne continue à produire des exclus et des inégalités sociales et du chômage, elle peut s'effondrer sous les coups de boutoir de démagogues nationalistes.

- La crise systémique mondiale (UL2). Les OIG ont échoué dans leur rôle de prévention. Pour un temps, elles sont hors jeu. Les FMN occupent le terrain libre et s'affirment plus que jamais comme les maîtresses du jeu. Ce sont elles qui essaient de réguler l'économie mondiale. Les États nationaux cèdent du terrain devant leurs exigences. Reste la société civile : ses organisations affaiblies tentent de se constituer en contre-pouvoir. Leur efficacité est fonction de la place qu'elles occupaient avant la crise, de la lucidité de leurs actions et de leur cohésion. Des conflits sporadiques éclatent un peu partout, révoltes et jacqueries se multiplient. Ces combats sont le plus souvent sans issue. Face à l'emprise des structures du réseau des multinationales, les syndicats de travailleurs s'unissent dans une centrale internationale.

- Un " autre " développement (GM2). Ce macro-scénario résulte de l'émergence de valeurs et d'exigences sociétales nouvelles et nombre de ces changements dans les mentalités collectives et individuelles se fraient déjà un chemin. Au niveau des objectifs, il ne s'agit plus de n'importe quelle croissance déterminée par le marché et l'emprise des FMN, mais d'un développement durable, responsable devant les générations futures, plus juste et moins inégalitaire. Tous les acteurs sont concernés : I'« autre » développement va du global au local et du local au global. Les OSNG, et plus généralement la société civile, en sont les concepteurs et les exécutants, les OIG mondiales et régionales, au sommet, sont des amplificateurs qui concrétisent les nouvelles règles du jeu, créent une pression de conformité qui oriente l'activité des FMN. Les États nationaux relaient ces exigences et entrent en compétition pacifique pour réaliser dans leur espace le mode de vie souhaité par leurs citoyens, pour eux-mêmes et pour leurs descendants.

- La crise régionale (GR2). Elle place les acteurs dans une position un peu différente de la crise mondiale. La modification tient aussi à la nature de la crise; nous avons retenu un fractionnement entre régions : I'OIG régionale est considérée comme un rempart défensif. L'État national recouvre une partie de sa souveraineté. Les OSNG exercent une forte influence à l'intérieur des territoires nationaux.

\section{L'articulation entre micro-et macro-scénarios : la prise en compte du temps}

Les micro-scénarios de la prospective protéines s'appuient sur un corps de soixante dix-neuf hypothèses considérées comme "internes " au système européen des protéines et qui ont été 
formulées à partir de l'analyse de ce système tel que nous l'avons perçu - les groupes d'experts et la cellule d'animation - au moment de l'élaboration des hypothèses, c'est-à-dire en 1998. Même si nous nous sommes efforcés de prendre du recul par rapport à cet environnement, ces hypothèses, et par conséquent les micro-scénarios qui en découlent, doivent s'interpréter de fait dans le cadre du contexte macro-économique de cette période. Cela ne signifie pas qu'il n'y a pas d'hypothèses de rupture au sein de ces hypothèses " internes " mais que ces dernières ont été envisagées ou retenues en ayant un cadre de représentation principalement déterminé par une configuration macroéconomique particulière.

En revanche, les macro-scénarios de cadrage mondial que nous venons de décrire, correspondent, par construction, à des images du futur et à une rupture par rapport au macro-scénario implicite de 1998. Dès lors, comment prendre en compte des évolutions du contexte macro-économique dans l'analyse de la dynamique du système des protéines ? La démarche proposée est d'actualiser le corps d'hypothèses internes et les relations entre hypothèses en se projetant à l'horizon temporel considéré et en choisissant un macro-scénario de référence, comme l'illustre le graphique. Les hypothèses sont actualisées - sélection, reformulation, identification de nouvelles hypothèses (portant sur des processus déjà en œuvre ou des processus nouveaux) - et le nouvel ensemble d'hypothèses peut être analysé de la même façon que précédemment.

Nous avons donc imaginé la mise en dynamique du système des protéines selon le processus itératif suivant :

- mettre en dynamique le jeu des hypothèses internes sous forme de micro-scénarios en nous plaçant implicitement dans le contexte de 1998, c'est-à-dire en considérant que le macro-scénario que nous avons implicitement pris en compte n'est pas remis en cause ;

- se projeter dans un horizon temporel suffisamment éloigné pour ouvrir le champ des possibles en termes de macro-scénario, autour de 2008, par exemple, après les négociations OMC, la nouvelle PAC et une situation probablement "décantée " dans le domaine des OGM, et réitérer l'exercice de prospective sur le système des protéines, à savoir produire de nouveaux corps d'hypothèses internes et construire des micro-scénarios pour chacun des macro-scénarios imaginés.

\section{L'actualisation du corps d'hypothèses}

La démarche de révision des micro-scénarios pour chacun des macro-scénarios n'a été qu'esquissée jusqu'à présent. Notre objectif premier étant d'évaluer la faisabilité de l'exercice d'actualisation, nous nous sommes limités à une analyse critique des soixante-dix-neuf hypothèses retenues et à la formulation d'une nouvelle liste d'hypothèses internes pour deux des six macro-scénarios : le toutlibéral (UL1) et la coopération entre blocs régionaux (GR1). La mise en œuvre complète de la démarche rentre désormais dans le cadre de l'appropriation et de la valorisation de ce travail par les opérateurs de la recherche.

Cette actualisation consiste à :

- Supprimer des hypothèses qui n'ont plus de sens dans le nouveau macro-scénario considéré parce que la configuration de ce dernier interdit d'envisager l'hypothèse, comme par exemple les hypothèses de la politique agricole commune pour les macro-scénarios qui ne prévoient aucune régulation à l'échelle régionale (UL1 par exemple). 
- Reformuler des hypothèses pour tenir compte de la nouvelle configuration du macro-scénario ou de l'évolution de l'horizon temporel. C'est le cas par exemple des hypothèses autour des OGM, qui ont été fortement déterminées par le contexte de 1998 alors que le débat évolue et que la problématique ne sera probablement pas du tout la même dans dix ans, quels que soient les choix qui seront effectués à court ou moyen termes.

- Ajouter de nouvelles hypothèses qui tiennent compte à nouveau des configurations particulières et du changement d'échelle temporelle, qui permet probablement d'envisager des ruptures plus fortes, notamment dans le secteur de la science et de l'innovation.

Une première actualisation a été effectuée pour UL1 et GR1, sans toutefois donner lieu à une analyse approfondie de la part de la cellule d'animation. D'autres hypothèses nouvelles sont envisageables, et c'est justement l'un des objectifs de la valorisation de la prospective que de permettre à l'utilisateur d'introduire ses propres hypothèses.

Dans le cas du macro-scénario UL1, trente hypothèses disparaissent, notamment celles concernant la Politique agricole commune tandis que neuf autres sont reformulées pour tenir compte de l'horizon temporel. Trente-six hypothèses sont maintenues et dix nouvelles apparaissent. Au total, la liste ne comporte plus que cinquante-quatre hypothèses.

Dans le cas de GR1, comme pour UL1, vingt-sept hypothèses sont abandonnées tandis que huit sont reformulées. Trente-huit ne sont pas modifiées et neuf nouvelles hypothèses sont formulées. La liste comporte cinquante-cinq hypothèses. Le tableau 3 donne des exemples d'actualisation d'hypothèses.

Les deux listes actualisées comportent beaucoup moins d'hypothèses que dans le macro-scénario de référence, ce qui résulte d'une part de l'abandon d'hypothèses jugées peu pertinentes et d'autre part, du cadrage plus étroit des macro-scénarios. Quarante-cinq hypothèses restent toutefois identiques aux deux listes, ce qui devrait simplifier l'analyse ultérieure.

Une fois le corps d'hypothèses modifié, la matrice de relations doit être actualisée, ce qui suppose de renseigner les nouvelles relations liées aux hypothèses introduites et d'actualiser les anciennes, puisque la relation entre deux hypothèses est renseignée pour un contexte donné et à un horizon temporel donné. L'analyse reste toutefois plus légère que pour la matrice initiale.

\footnotetext{
Encadré

Quelques sorties des différents macro-scénarios de cadrage mondiaux sur le système des protéines

Nous présentons, par macro-scénario, quelques-unes des "sorties ", des conséquences... sur le système des protéines.

\section{Macro-scénario UL1 : Le tout-libéral}

* Sous l'effet combiné de la croissance démographique dans les principaux pays en développement (et notamment la Chine), de l'accroissement des revenus par tête et de la constitution d'une classe
} 
moyenne surtout urbaine, la consommation mondiale de viande augmente très fortement, surtout sous forme de porcs et de volailles.

* La poursuite de la libéralisation des échanges mondiaux, notamment en termes de viande (particulièrement au Japon, dans les pays émergents d'Asie, en Chine et en Russie), permet un fort accroissement du commerce mondial des viandes, qui bénéficie en particulier aux États-Unis et à I'UE.

* Les faibles contraintes environnementales sur les productions animales aux États-Unis et dans I'UE permettent de poursuivre l'intensification des productions animales et de maintenir de faibles coûts de production (hors aliment). Les investissements des grandes firmes multinationales dans ces deux zones, en trituration et aliments composés, sont très importants.

* Les progrès de la génétique végétale sont importants et rapidement mis en œuvre aux États-Unis, dans I'UE et en Amérique du Sud sur les trois principales cultures (maïs, soja et blé) et entraînent un différentiel de rendement croissant par rapport aux autres cultures (riz, colza, tournesol, autres céréales) et aux productions des pays en développement.

* La mondialisation des systèmes de consommation alimentaire est très forte, grâce en particulier au développement de la grande distribution, et la part des protéines animales augmente fortement dans l'ensemble du monde par rapport aux protéines végétales.

* Le modèle maïs-soja d'alimentation animale domine, et la priorité est donnée à l'abaissement des coûts de production.

\section{Macro-scénario UL2 : La crise systémique mondiale}

* La croissance économique mondiale est faible (notamment au Japon, dans les pays émergents d'Asie, en Amérique du Sud et en Russie), et la consommation de viande par tête progresse moins vite. Elle continue cependant à augmenter en tonnage, à cause de l'effet démographique.

* Comme en UL1, le rôle des politiques agricoles dans les pays développés comme dans les pays en développement (subventions aux exportations, aides à la production, contrôles des importations) diminue fortement et déstabilise la production agricole dans de nombreux pays en développement faute d'une protection suffisante.

* Les grandes firmes multinationales privilégient les investissements directs dans le secteur de la trituration, des aliments composés et de l'élevage dans les pays à fort potentiel de croissance de la demande de viande ou de rationalisation de celle-ci.

* En l'absence de fortes contraintes environnementales, les productions animales s'intensifient en adoptant largement le modèle maïs-soja.

* Face à une faible croissance de la production végétale dans des pays qui ne bénéficient pas de l'apport des nouvelles technologies de la génétique et qui sont pénalisés par des problèmes d'irrigation et de disponibilité en terre (sauf en Amérique du Sud), les importations de protéines végétales se développent fortement, surtout sous forme de maïs et de soja. 


\section{Macro-scénario GM1 : La régulation mondiale}

* La libéralisation des échanges mondiaux se stabilise au niveau des engagements pris dans le cadre de l'Uruguay Round et de I'OMC et la politique des États-Unis se poursuit dans le prolongement du Fair Act.

* Les politiques agricoles régionales sont compatibles avec la gouvernance mondiale.

* Les OIG interviennent de façon plus importante dans le cadre de l'aide alimentaire sur les protéines végétales. Celle-ci s'effectue sous forme monétaire, ce qui permet un certain dynamisme des exportations des États-Unis.

* L'OMS coordonne les recherches sur la santé et diffuse des normes nutritionnelles.

Macro-scénario GM2 : Un « autre » développement

* Les préoccupations environnementales sont beaucoup plus fortes et sont fortement prises en compte aussi bien dans les politiques des États-Unis et de I'UE que dans celles des OIG.

* Ces réglementations se traduisent par une croissance plus lente des rendements des produits végétaux dans les pays développés et par une aide de la part des OIG dans le transfert des nouvelles technologies aux pays en développement, diminuant ainsi l'écart des rendements entre pays développés et pays en développement pour les principales cultures (maïs, soja, blé) et entre ces cultures et les autres (riz, céréales secondaires, autres oléagineux).

* Les consommateurs, particulièrement dans les pays développés, sont de plus en plus soucieux des problèmes de santé, de qualité et de traçabilité. L'attrait de la viande diminue au profit des protéines végétales équilibrées.

* Les contraintes réglementaires sur les rejets et sur le bien-être animal augmentent fortement les coûts de production des systèmes intensifs (porcs, volailles) et favorisent l'émergence de productions plus extensives et plus diversifiées, avec une forte augmentation des marchés adaptés à des besoins spécifiques (segmentation et différenciation des prix).

* Les OIG autorisent une plus grande liberté aux États pour définir les critères de " qualité " des produits alimentaires d'importation et éventuellement refuser les produits obtenus par certaines technologies (OGM, clonage, etc.).

\section{Macro-scénario GR1 : La coopération entre blocs}

* En matière agricole, la guerre commerciale entre l'ALENA et l'UE est évitée : un modus vivendi régit les échanges agricoles entre les États-Unis, l'Argentine, le Brésil, I'Australie et I'UE sur la base d'avantages réciproques. Des politiques agricoles se développent à l'intérieur de chaque zone régionale ; le commerce agricole à l'intérieur de ces zones progresse fortement.

* Afin de pallier une chute des prix mondiaux des produits de grande culture (céréales et oléagineux), l'ALENA et l'UE mettent en place des systèmes de contrôle quantitatif des productions 
(gels de terres) et se répartissent les marchés extérieurs en privilégiant les exportations de produits à forte valeur ajoutée (viande, produits laitiers), en laissant une place plus grande à l'exportation des produits végétaux en provenance d'Amérique du Sud et d'Australie.

* L'ouverture des marchés extérieurs à I'ALENA et à l'UE est obtenue comme contrepartie à une forte aide économique et financière.

\section{Macro-scénario GR2 : La crise régionale}

* Le commerce mondial des produits agricoles entre grandes zones diminue fortement, aussi bien pour les produits végétaux que pour la viande.

* Les spécialisations internes des activités ne sont plus induites par la seule compétitivité, mais par le quasi-enfermement obligé.

* Les systèmes de protection à l'entrée de ces zones (barrières tarifaires et non tarifaires) se développent, et chaque zone recherche une utilisation optimale des matières premières disponibles localement.

* Les modes d'alimentation des animaux sont très diversifiés et s'éloignent du modèle maïs-soja.

* Les besoins alimentaires de certaines zones, coupées du commerce mondial, sont de moins en moins bien satisfaits, et les consommations de protéines animales par tête diminuent dans certains cas.

Notes :

${ }^{1}$ Actuellement, directeur scientifique du Centre technique interprofessionnel des oléaginaux métropolitains (CETIOM).

${ }^{2}$ Actuellement, adjointe au sous-directeur de la Qualité et de la Protection des végétaux, ministère de l'Agriculture.

${ }^{3}$ Ce qui ne signifie pas qu'il n'y ait pas de prise en compte du climat dans les politiques.

${ }^{4}$ Nous nous inspirons ici de la typologie proposée par Kimon Valaskakis [2].

${ }^{5}$ Sur la notion de « soutien » dans l'analyse systémique de la politique, voir [4]. ${ }^{6}$ Dans le rapport publié à l'issue de cette prospective, P. Gonod a réactualisé les macro-scénarios jusqu'en début 2001. À cette époque, la conclusion était que le contexte mondial restait polymorphe, sans qu'aucun macroscénario ne semble clairement devoir s'imposer [5].

\section{CONCLUSION}

Les macro-scénarios sont nés de la nécessité de prendre en compte les évolutions macro-économiques du contexte mondial dans l'analyse de la dynamique du système européen des protéines. Un cadre méthodologique a été proposé afin d'articuler les micro-scénarios et les macro-scénarios et pourra être mis en œuvre dans le cadre de la valorisation en cours de la prospective protéines. Toutefois, les macro-scénarios, tels qu'ils ont été esquissés ici, ont une portée beaucoup plus générique et pourraient constituer un cadre de réflexion pour d'autres exercices de prospective. 
Il faut toutefois souligner que l'objectif ne saurait être de réaliser une prospective à l'échelle macroéconomique mondiale ni de s'intéresser aux transitions possibles entre macro-scénarios. Leur robustesse pourra être appréciée par le lecteur dans la mesure où la description qui en est faite ici n'a pas été modifiée depuis leur élaboration en 1998. Les nombreux événements survenus depuis cette époque nous semblent avoir modifié sensiblement le contexte macro-économique, sans remettre en cause fondamentalement l'éventail des futurs possibles proposé par les six macro-scénarios retenus ${ }^{6}$.

\section{REFERENCES}

1. MESSEAN A, LECEUUR H, SEBILLOTTE M (2001). Prospective : les protéines végétales et animales. Enjeux de société et défis pour l'agriculture et la recherche. Éditions INRA, collection Bilan et Prospectives, Paris.

2. VALASKAKIS K (1998). Mondialisation et gouvernance. Futuribles, $n^{\circ} 230$, pp. 5-28.

3. PERROUX F (1975). Unités actives et mathématiques nouvelles. Révision de la théorie de l'équilibre économique général. Dunod, Paris.

4. EASTON D (1965). A system analysis of political life. John Wiley \& Sons, Inc, New York.

5. GONOD P (2001). L'évolution de la configuration prospective. In : Messean A, Lecœur H, Sebillotte $M$. Prospective : les protéines végétales et animales. Enjeux de société et défis pour l'agriculture et la recherche. Éditions INRA, collection Bilan et Prospectives, Paris.

Illustrations

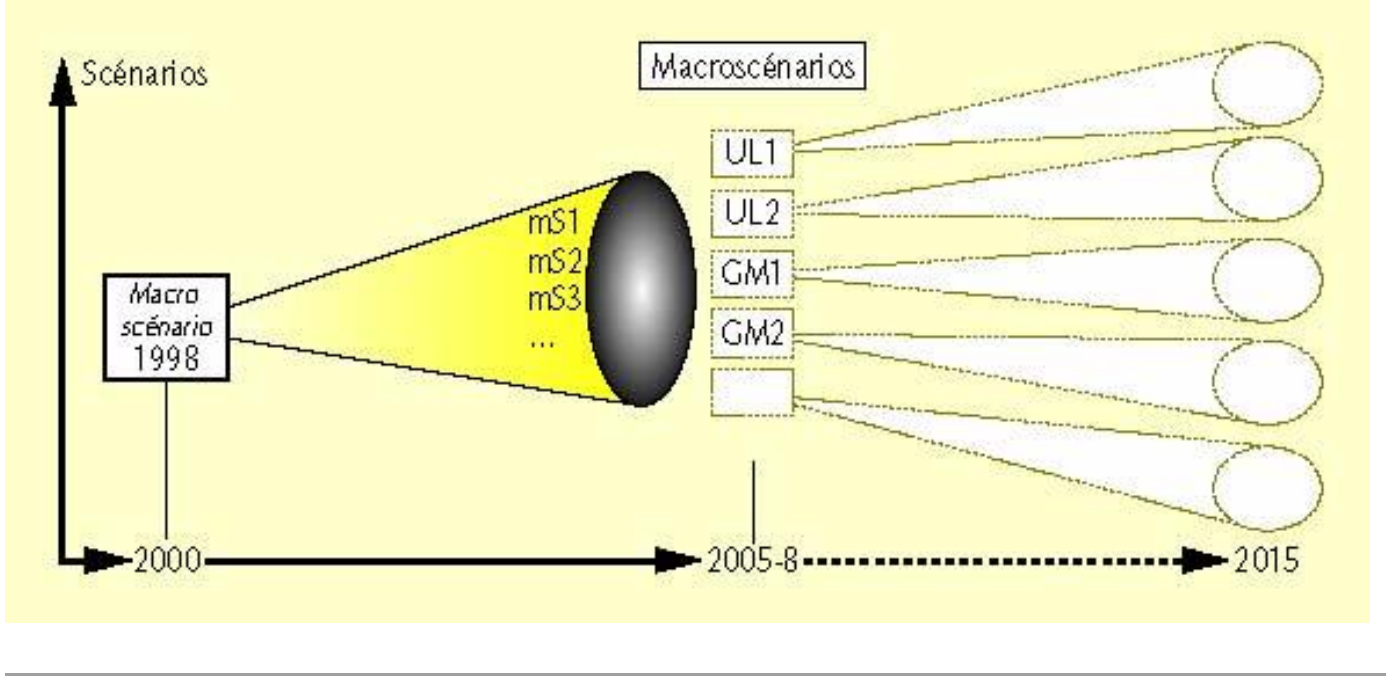

Graphique 1. Micro-scénarios et macro-scénarios : leur articulation dans le temps 
Tableau 1. Typologie des macro-scénarios.

\begin{tabular}{|c|c|c|}
\hline Macro-scénarios & \multicolumn{2}{|c|}{ Variantes } \\
\hline Ultra-libéralisme & $\begin{array}{c}\text { UL1 } \\
\text { * Le to ut-libéral } \%\end{array}$ & $\begin{array}{c}\text { UL2 } \\
\text { * La aise systémique } \\
\text { mondiale * }\end{array}$ \\
\hline $\begin{array}{l}\text { Gouvernance } \\
\text { mondiale }\end{array}$ & $\begin{array}{c}\text { GM1 } \\
\text { * La régulation mondiale } \\
\text { par les organisctions } \\
\text { intergouver nementales * }\end{array}$ & $\begin{array}{l}\text { GM2 } \\
\text { Un* autre * } \\
\text { développement }\end{array}$ \\
\hline $\begin{array}{l}\text { Gouvernances } \\
\text { régionales }\end{array}$ & $\begin{array}{c}\text { GR1 } \\
\text { * La coopération entre } \\
\text { gandes zones } \\
\text { économiques mondiales * }\end{array}$ & $\begin{array}{c}\text { GR2 } \\
\text { * Les aises régio nales * }\end{array}$ \\
\hline
\end{tabular}

Tableau 2. Positionnement des acteurs par rapport aux mađo-scénarios.

\begin{tabular}{|c|c|c|}
\hline Macro-scén arios & \multicolumn{2}{|c|}{ Variantes } \\
\hline Libéralisme & $\begin{array}{l}\text { UL1: Le tout-libéral } \\
\text { Les FMN sont } \\
\text { maîtresses du jeu } \\
\text { Les EN, OICM, OICR } \\
\text { cèdent du terrain } \\
\text { Les OSNG sont } \\
\text { sans pouvoir }\end{array}$ & $\begin{array}{l}\text { UL2: La a ise systémique mondiale } \\
\text { Les FMN restent } \\
\text { maîtresses du jeu } \\
\text { Les EN et OIGR cèdent du terrain } \\
\text { Les OICM ont échoué } \\
\text { Les OSNG sont sans réel pouvoir }\end{array}$ \\
\hline $\begin{array}{c}\text { Gouvernance } \\
\text { mondiale }\end{array}$ & $\begin{array}{l}\text { GM1: La régulation } \\
\text { par les OKM } \\
\text { Les OICM gagnent } \\
\text { du pouvoir }\end{array}$ & $\begin{array}{l}\text { GM2: Un autre développement } \\
\text { Les FMN perdent de l'in fluence } \\
\text { Les OSNG sont les conceptrices } \\
\text { des valeurs nouvelles } \\
\text { Les EN, OIGR et OIGM } \\
\text { ont un rôle accru }\end{array}$ \\
\hline $\begin{array}{l}\text { Gouvernance } \\
\text { régionale }\end{array}$ & $\begin{array}{l}\text { GR1: La coopération } \\
\text { entre blocs } \\
\text { Les OIGR ont } \\
\text { une forte influ ence }\end{array}$ & $\begin{array}{c}\text { GR2: La } \sigma \text { iserégionale } \\
\text { Les OIGR ont une influen ce } \\
\text { prim ordiale dans le cadre } \\
\text { du fractionnement entre régions }\end{array}$ \\
\hline
\end{tabular}

EN : etats nationaux; HMN : firmes multinationales ; UKM: orqanismes interqouvernementaux mondiaux; OKR : orqanis mes interqouve rnementaux réqioraux ; OSNG : orqa nisatio rs sociales non qouvernementales; OPA : orqanisations profession nelles aqricoles. 
Tableau 3. Exemple d'actualisction d'hypothèses.

\begin{tabular}{|c|l|c}
\hline Macro-scénarios & \multicolumn{1}{|c|}{ Hypoth èse } & Statut \\
\hline \multirow{5}{*}{ UL1 } & $\begin{array}{l}\text { L'Ut maintient une politique d'encou- } \\
\text { ragement spécifiqueaux protéagineux } \\
\text { qui peuvent ainsi se maintenir. }\end{array}$ & Abandonnée \\
\cline { 2 - 3 } & $\begin{array}{l}\text { Les FMN proposent des modèles de } \\
\text { con som ma tion génériques déc linés } \\
\text { régionalement selon des composantes } \\
\text { culturelles. }\end{array}$ & Reformulée \\
\cline { 2 - 4 } & $\begin{array}{l}\text { Le ranching apparait au sein de l'Utet } \\
\text { remplace progressivement les systèmes } \\
\text { de production de troupeaux allaitants. }\end{array}$ & Nouvelle \\
\hline \multirow{2}{*}{ GRI } & $\begin{array}{l}\text { Les consomma teurs contribuent, par } \\
\text { leurschoix, au maintien de filères non } \\
\text { OGM significatives. }\end{array}$ & Abandonnée \\
\cline { 2 - 4 } & $\begin{array}{l}\text { Les Etats-Unis et l'UE harmonisent leur } \\
\text { législation da ns le doma ine du droit } \\
\text { des brevets. }\end{array}$ & Reformulée \\
\cline { 2 - 4 } & $\begin{array}{l}\text { Des pois à haute teneur en protéines } \\
\text { sont mis au point. }\end{array}$ & Nouvelle \\
\hline
\end{tabular}

